\title{
Data Mining based Prediction of Unemployment Rate Data by using Radial Basis Function Neural Network (RBFNN)
}

\author{
${ }^{1}$ Younis Kadthem Hameed, ${ }^{2}$ Wahab S. Mohammed and ${ }^{1}$ Fadhel Kadhem Zaidan \\ ${ }^{1}$ Department of Statistic, College of Administration and Economics, \\ ${ }^{2}$ Electronic Computing Center, University of Diyala, Baqubah, Iraq \\ mscyounis@gmail.com
}

\begin{abstract}
Data mining is one of the knowledge discovery steps in database in which modeling techniques are applied. In this study, the unemployment rate in the Iraqi database prediction model is determined according to the RBF neural network where the principle and algorithm of the RBF is used. Our study also predicts the future unemployment rate in Iraq. To increase the efficiency of mining process, some pre-processing needs to be done to the data. Experimental results showed the good accuracy when applied to the adjust data.
\end{abstract}

Key words: Radial basis function, predict, data mining, database, predicts, mining process

\section{INTRODUCTION}

The forecasting methods which are all around embraced have the accompanying sorts: basic relapse examination strategy (SRA), Exponential Smoothing strategy (ES), complete autoregressive versatile investigation strategy (CAMA) and so on. These strategies are great connected in a few expectations, however, regardless they exist numerous deformities These techniques have a decent impact of straight forecast, yet are not extremely exact of nonlinear expectation these strategies don't matter to multi-factor conjecture the foundation of the forecast show is intensely reliant on the information level of the anticipate individuals. Manufactured neural system (ANN) displaying it's one of successful technique to investigation strategies for gauging which can well uncover the connection of nonlinear time arrangement in defer state space. Then, the ANN can accomplish the motivation behind expectation. The Kolmogorov continuation hypothesis in neural system hypothesis guarantees the expectation possibility of the neural system which is utilized for time arrangement from the perspective of arithmetic. The joblessness rate is chosen by numerous goal factors. The conjecture of the joblessness rate has not great ways now. Because the joblessness rate has a decent nonlinear trademark, the best use of the RBF neural system to deal with nonlinear issues, then the RBF neural system can be applied to estimate the joblessness rate. The study sets up forecast demonstrate and predicts the joblessness rate. The after effect of trial demonstrates that the forecast display has great expectation impact. Joblessness in Iraq has turned into a financial and social measurement of the issue of plainly communicating deficiency in monetary structures and deformity social at the national level where joblessness is a genuine social scourge of human limit crashes and scattered development openings monetary prosperity. In order to monitor the level of unemployment trends in Iraq it must provide periodic statistical indicators reflect the reality of the size of this phenomenon. It has been taking the unemployment rate in Iraq from 1990-2013 (Wei, 2005).

Radial Basis Function (RBF) networks: RBF systems are bolster forward systems prepared utilizing a managed preparing calculation. They are sometimes arranged with a solitary shrouded layers of the units whose enactment work is chosen from class of capacities called premise capacities. While like back spread in numerous regards, spiral premise work systems have a few favorable circumstances. They as a rule prepare substantially quicker than back proliferation systems. They are less powerless to issues with non-stationary information sources due to the conduct of the spiral premise work concealed units.

Popularized by Moody and Darken RBF networks have proven to be useful neural network architecture. The real distinction between RBF arranges and back proliferation organizes (that is multi-layer perceptron prepared by back spread calculation) is the conduct of the single concealed layer. Instead of utilizing the sigmoidal

Corresponding Author: Younis Kadthem Hameed, Department of Statistic, College of Administration and Economics, University of Diyala, Baqubah, Iraq, mscyounis@gmail.com 
or S-molded actuation study as in back engendering, the concealed units in RBF systems utilize a Gaussian or some different premise piece work. Each concealed unit goes about as a privately tuned processor that registers a score for the match between the information vector and its association weights or focuses. In actuality, the premise units are exceptionally specific example finders. The weights interfacing the premise units to the yields are utilized to take straight mixes of the shrouded units to item the last characterization or yield (Sun et al., 2002).

\section{MATERIALS AND METHODS}

The RBF networks structure: Radial basis function network has 3-layers feed-forward neural network which incorporates an info layer, a shrouded layer with outspread premise work neurons and a yield layer with direct neurons as shown in Fig. 1. Concealed layer is generally utilizing spiral premise work as excitation work and the outspread premise excitation work is normally Gaussian capacity which is typically communicated as:

$$
R\left(x^{\mathrm{q}}-\mathrm{c}_{\mathrm{i}}\right)=\exp \left[-\left(\left\|\mathrm{w} 1_{\mathrm{i}}-\mathrm{x}^{\mathrm{q}}\right\| \times \mathrm{bl} 1_{\mathrm{i}}\right)^{2}\right]
$$

where, $\left\|\mathrm{w} 1_{1}-\mathrm{x}^{\mathrm{q}}\right\|$ is the Euclidean distance, $\mathrm{c}$ is the center of Gaussian function. $\mathrm{X}^{\mathrm{q}}=\left\{\mathrm{X}_{1}^{\mathrm{q}}, \mathrm{X}_{2}^{\mathrm{q}}, \ldots, \mathrm{X}_{1}^{\mathrm{q}}\right\}$ is the $\mathrm{qth}$ to input data. $w l_{i}$ it isthe distance between weight vector, associated with the contributing layer and in each neuron in the shrouded layer, the info vector is $\mathrm{x}^{\mathrm{q}}$ increased by the limit $b 1_{i}$ which is considered as its own particular information asshown in Fig. 2 (Sun et al., 2002). So, we get the ith input of hidden layer neuron which can be expressed as $\mathrm{k}_{\mathrm{i}}^{\mathrm{q}}$ :

$$
\mathrm{k}_{\mathrm{i}}^{\mathrm{q}}=\sqrt{\sum_{\mathrm{j}}\left(\mathrm{w} 1_{\mathrm{ji}}-\mathrm{x}_{\mathrm{j}}^{\mathrm{q}}\right)^{2}} \times \mathrm{bl}
$$

ith output of hidden layer neuron can be expressed as $\mathrm{r}_{1}^{\mathrm{q}}$ :

$$
\mathrm{r}_{\mathrm{i}}^{\mathrm{q}}=\exp \left[\sqrt{\sum_{\mathrm{j}}\left(\mathrm{w} 1_{\mathrm{ji}}-\mathrm{x}_{\mathrm{j}}^{\mathrm{q}}\right)^{2}} \times \mathrm{bl} \mathrm{i}_{\mathrm{i}}\right]
$$

The RBF neural network output is the weighted summation of each concealed layer neuron's yield and the excitation work is utilizing unadulterated straight capacity, so the qth yield layer neuron's yield which is comparing to theqth input can be expressed as $y^{q}$ :

$$
\mathrm{y}^{\mathrm{q}}=\sum_{\mathrm{i}=1}^{\mathrm{n}} \mathrm{r}_{\mathrm{i}}^{\mathrm{q}} \times \mathrm{w} 2_{\mathrm{i}} \mathrm{h}
$$

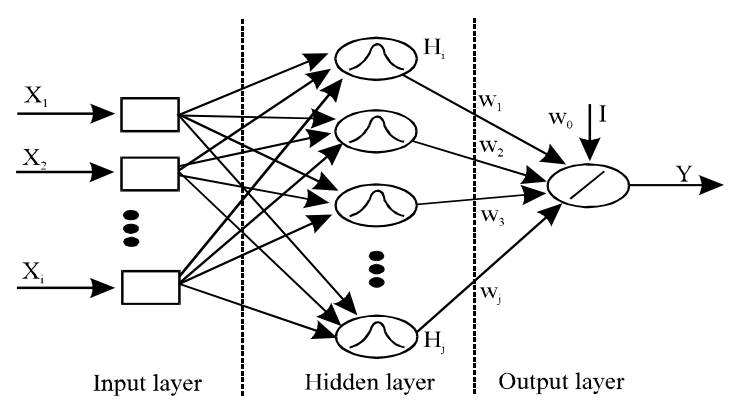

Fig. 1: Note how the inscription is focused in the segment

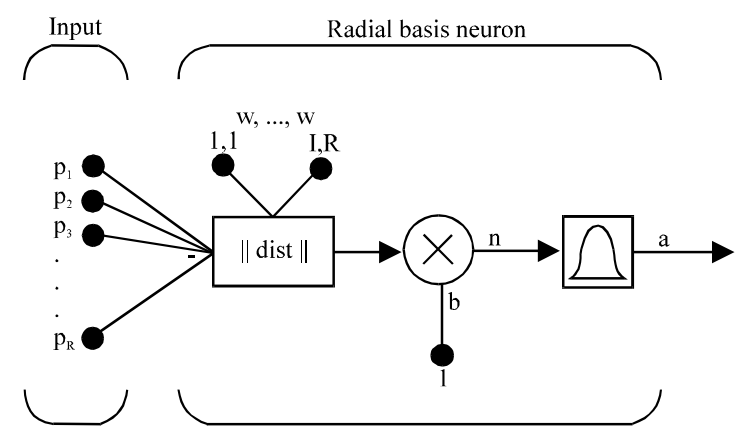

Fig. 2: RBF neural system input and output of shrouded layer neurons

Radial basis function network algorithm: RBF neural network learning procedure can be partitioned into $h(x)$ is the Gaussian initiation work with the parameters $r$ (the span or standard deviation) and $\mathrm{c}$ (the middle or normal taken from the information space) characterized independently at each RBF unit. The learning procedure dependon modifying the parameters of the system to duplicate an arrangement of information yield designs. There are three sorts of parameters the weight $\mathrm{w}$ between the shrouded hubs and the yield hubs, the middle $\mathrm{c}$ of every neuron of the concealed layer and the unit width r (Sun et al., 2002).

Unit center (c): Any bunching calculation can be utilized to decide the RBF unit centers (e.g., k-means clustering). An arrangement of groups each with r-dimensional focuses is dictated by the quantity of info factors or hubs of the information layer. The group focuses turn into the focuses of the RBF units. The quantity of bunches, $\mathrm{H}$ is a plan parameter and decides the quantity of hubs in the shrouded layer. The k-implies bunching calculation continues as follows: initialize the focal point of each group to an alternate arbitrarily chose preparing design. Assign each preparation example to the closest group. This can be expert by computing the Euclidean 
Table 1: The unemployment rate in Iraq on 1990-2013

\begin{tabular}{lc}
\hline Years & Unemployment rate \\
\hline 1990 & 5.5 \\
1991 & 6.5 \\
1992 & 7.5 \\
1993 & 8.5 \\
1994 & 10.5 \\
1995 & 12.9 \\
1996 & 13.9 \\
1997 & 15.4 \\
1998 & 17.4 \\
1999 & 20.2 \\
2000 & 22.4 \\
2001 & 24.6 \\
2002 & 26.7 \\
2003 & 28.1 \\
2004 & 26.8 \\
2005 & 17.9 \\
2006 & 17.5 \\
2007 & 11.7 \\
2008 & 15.3 \\
2009 & 14 \\
2010 & 12 \\
2011 & 11 \\
2012 & 11.9 \\
2013 & 12.1 \\
\hline $09 t a$ &
\end{tabular}

Data from the unemployment rate in Iraq of central statistical organization

separations between the preparation designs and the bunch focuses. When all preparation designs are appointed, figure the normal position for each group focus. They at that point turn out to be new bunch focuses. Repeat stages 2 and 3, until the point that the group focuses don't change amid the consequent cycles (Table 1).

Unit width (r): At the point when the RBF focuses have been built up, the width of each RBF unit can be ascertained utilizing the $\mathrm{k}$-closest neighbor's calculation. A number $\mathrm{k}$ is picked and for each middle, the $\mathrm{k}$ closest focuses is found. The root-mean squared separation between the present bunch focus and its $\mathrm{k}$ closest neighbors is ascertained and this is the esteem decided for the unit width (r). So, if the current cluster center is $c_{j}$, the $\mathrm{r}$ value is:

$$
r_{j}=\sqrt{\frac{\sum_{i=1}^{k}\left(c_{j}-c_{i}\right)^{2}}{k}}
$$

Variance (ס): The basis function of RBF neural network is Gaussian function, therefore, variance can be given as:

$$
\sigma_{i}=\frac{c_{\max }}{\sqrt{2 h}} i=1,2,3, \ldots, h
$$

$\mathrm{c}_{\max }$ is maximum distance among the selected centers.
Weights (w): The weights associating the shrouded layer to produce a layer of neurons can be obtained specifically through the lowest square technique. Its computation recipe is as follows:

$$
\mathrm{W}=\exp \left[\mathrm{c}_{\max }^{\frac{\mathrm{h}}{2}\left\|\mathrm{x}_{\mathrm{q}}-\mathrm{c}_{\mathrm{i}}\right\|^{2}}\right]
$$

Input samples pretreatment: Since, the verifiable capacity of RBF neural system is Gaussian capacity in general requires for input an incentive in the vicinity of 0 and 1, do standardize on the joblessness rate in Iraq from 1990-2013. Standardization is fundamentally a similar method to factual information standardization, for the most part utilizing the accompanying structure:

$$
\overline{\mathrm{X}}=\frac{\mathrm{X}-\mathrm{X}_{\min }}{\mathrm{X}_{\max }-\mathrm{X}_{\min }}
$$

where, $\mathrm{X}$ is the genuine estimation of test, $\mathrm{X}_{\max }$ takes an extensive esteem, guaranteeing conjecture year is not as much as the esteem; $\mathrm{X}_{\min }$ takes an example of information is not as much as the base an incentive to guarantee standardized esteem isn't near 0 . After the pretreatment of information finishes the preparation, due process information (backwards change) to get the real esteem (Liu et al., 2009).

\section{RESULTS AND DISCUSSION}

The quantity of info layer neurons comparing to the measurement of information vectors, the quantity of information layer hubs is excessively, making the system take in the quantity of moderately expansive input hubs is nearly nothings, follow-up values can't mirror the estimation of the relationship between the forerunner. That leads to decide, the quantity of information neuron of RBF neural system is 5 and the quantity of yield neurons is 1. Treating the samples as follows: Input neuron $\mathrm{P}=[\mathrm{p}(\mathrm{t}-5), \mathrm{p}(\mathrm{t}-4), \mathrm{p}(\mathrm{t}-3), \mathrm{p}(\mathrm{t}-2), \mathrm{p}(\mathrm{t}-1)]$ output neurons $\mathrm{T}=\left[\mathrm{p}^{\prime}(\mathrm{t})\right]$. Where, $\mathrm{t}=1990,1991, \ldots, 2013, \mathrm{P}(\mathrm{t})$ denote the normalized number of unemployment rate at $t$ year. We can obtain in this method the training samples and test samples as shown in Table 2. To test the accuracy and efficiency of the network, select group 1-18 group data as the study samples, the groups (19-24) as the test samples and using the trained RBF neural networks to predict (Zhang and Li, 2012). Creating a precise neural network by new be function, this function 
Table 2: The train and test group actual unemployment rate in Iraq on 1990-2013

\begin{tabular}{|c|c|c|c|c|c|c|}
\hline \multirow[b]{2}{*}{ Groups } & \multicolumn{5}{|c|}{ Input neurons $P=[p(t-5), p(t-4), p(t-3), p(t-2), p(t-1)]$} & \multirow{2}{*}{$\begin{array}{l}\text { Output neurons T } \\
p^{\prime}(t)\end{array}$} \\
\hline & $p(t-5)$ & $p(t-4)$ & $p(t-3)$ & $p(t-2)$ & $p(t-1)$ & \\
\hline \multicolumn{7}{|c|}{ Train group } \\
\hline 1 & 0.194567 & 0.987435 & 0.950681 & 0.892438 & 0.808902 & 0.208798 \\
\hline 2 & 0.987435 & 0.809456 & 0.987435 & 0.950681 & 0.878696 & 0.246352 \\
\hline 3 & 0.950681 & 0.987435 & 0.342334 & 0.987435 & 0.934967 & 0.287871 \\
\hline 4 & 0.892438 & 0.950681 & 0.987435 & 0.242325 & 0.974468 & 0.333158 \\
\hline 5 & 0.771664 & 0.853805 & 0.921099 & 0.968884 & 0.883489 & 0.414091 \\
\hline 6 & 0.603957 & 0.697601 & 0.785644 & 0.862703 & 0.965637 & 0.516276 \\
\hline 7 & 0.509827 & 0.603957 & 0.697601 & 0.785644 & 0.922668 & 0.573778 \\
\hline 8 & 0.394785 & 0.482695 & 0.575443 & 0.668881 & 0.843033 & 0.649555 \\
\hline 9 & 0.272562 & 0.346139 & 0.428602 & 0.517458 & 0.71962 & 0.740945 \\
\hline 10 & 0.152859 & 0.203679 & 0.264616 & 0.335199 & 0.540095 & 0.847642 \\
\hline 11 & 0.087347 & 0.121191 & 0.163951 & 0.216259 & 0.399033 & 0.918281 \\
\hline 12 & 0.046359 & 0.066978 & 0.09435 & 0.129591 & 0.277529 & 0.967183 \\
\hline 13 & 0.023476 & 0.035273 & 0.051674 & 0.073812 & 0.184786 & 0.990379 \\
\hline 14 & 0.013602 & 0.021067 & 0.031815 & 0.046845 & 0.132488 & 0.993535 \\
\hline 15 & 0.01645 & 0.025382 & 0.038185 & 0.056012 & 0.150833 & 0.016358 \\
\hline 16 & 0.091213 & 0.127358 & 0.173386 & 0.230154 & 0.402869 & 0.824165 \\
\hline 17 & 0.079751 & 0.112202 & 0.153917 & 0.205866 & 0.368498 & 0.803989 \\
\hline 18 & 0.126172 & 0.167059 & 0.215672 & 0.271479 & 0.41411 & 0.56472 \\
\hline \multicolumn{7}{|c|}{ Test group } \\
\hline 19 & 0.070261 & 0.098601 & 0.134917 & 0.179998 & 0.322061 & 0.699474 \\
\hline 20 & 0.064631 & 0.090357 & 0.123169 & 0.163703 & 0.29311 & 0.634265 \\
\hline 21 & 0.061057 & 0.084287 & 0.113451 & 0.148892 & 0.262939 & 0.540698 \\
\hline 22 & 0.050829 & 0.070168 & 0.094446 & 0.12395 & 0.223983 & 0.486746 \\
\hline 23 & 0.036194 & 0.05118 & 0.070564 & 0.094859 & 0.185246 & 0.501795 \\
\hline 24 & 0.026788 & 0.038459 & 0.053835 & 0.073477 & 0.152504 & 0.488568 \\
\hline
\end{tabular}

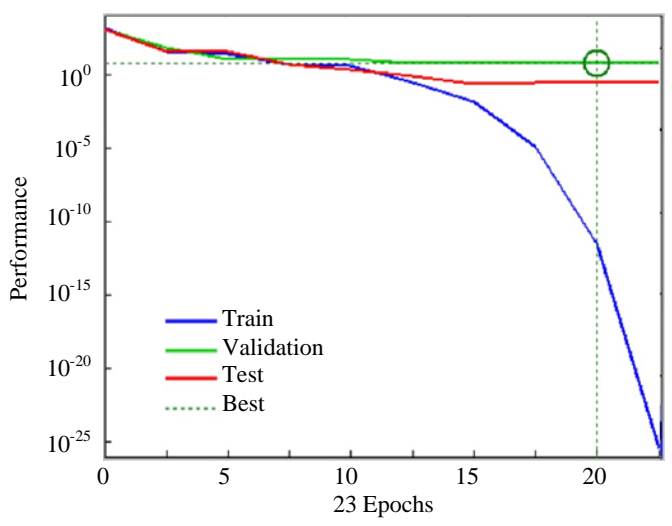

Fig. 3: The result of RBF neural network training in 23 epochs; Performance is $1.02234 \mathrm{e}-025$, Goal is $1 \mathrm{e}-005$

creates RBF network, automatically select the number of hidden layer and make the error to 0 , training sample, shown in Fig. 3. Using MATLAB (Defeng, 2009a, b). In this Fig. 3, the percentage of training is $60 \%$, validation is $20 \%$ and test is $20 \%$ in 23 epochs. Then we can get the $\mathrm{RBF}$ neural network learning and training curve as shown in Fig. 4. Now, we can be testing the neural network and verifying the prediction performance, shown in Fig. 5. This gives an exact premise to foreseeing joblessness rate in Iraq later on. Enter the genuine incentive from 2005-2013 can get the standardized

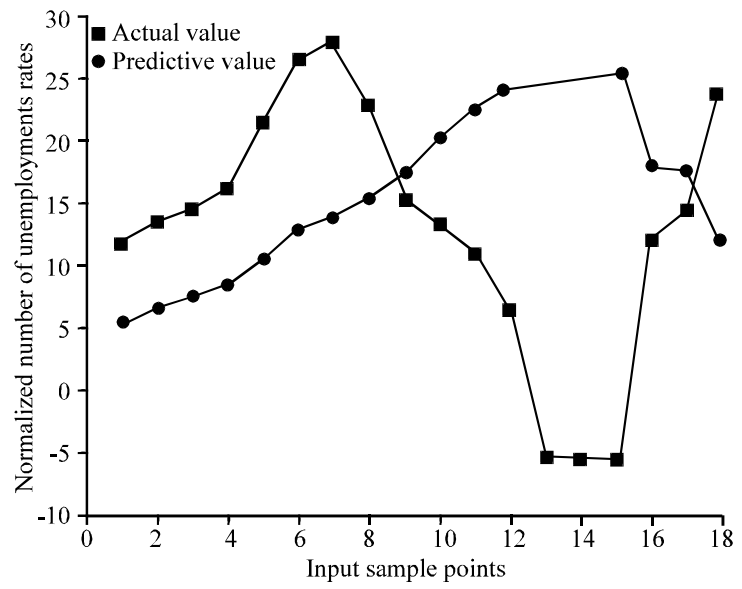

Fig. 4: RBF neural network learning and training curve

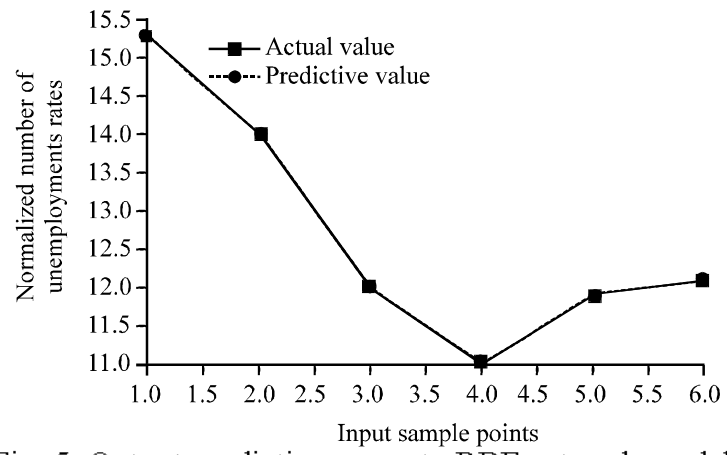

Fig. 5: Output prediction curve to RBF network model 
Table 3: The predicted value of unemployment rate in Iraq from 20142023

\begin{tabular}{lc}
\hline Years & Predictive values \\
\hline 2014 & 12.7 \\
2015 & 14.5 \\
2016 & 23.5 \\
2017 & 23.9 \\
2018 & 28.1 \\
2019 & 24.6 \\
2020 & 22.4 \\
2021 & 24.3 \\
2022 & 24.8 \\
2023 & 26.5 \\
\hline
\end{tabular}

anticipated an incentive in 2014. Similarly, after a multi-step iterative can get the standardized anticipated an incentive from 2014-2023, at that point after backwards change, the anticipated esteem can be gotten from 2014-2023 as shown in Table 3 (Defeng, 2009a, b; Sing et al., 2003).

\section{CONCLUSION}

This study has introduced an information mining of joblessness rate in Iraq forecast strategy in view of RBF neural system. Through RBF neural system versatile, self-association and self-learning capacity, influencing joblessness to rate like clockwork as the RBF neural system's information, we can anticipate joblessness rate in the ten year. After MATLAB preparing, estimating, reenactment, this technique accomplishes a decent expectation impact. The utilization of this technique gives another state of mind for mimicking and predicting unemployment rate Iraq.

\section{REFERENCES}

Defeng, Z., 2009a. MATLAB Neural Network Application Design. Mechanical Industry Press, Beijing, Chaina,.

Defeng, Z., 2009b. MATLAB Neural Network Simulation and Application. Electronic Industry Press, Beijing, China,

Liu, X.Q., X.Y. Wang and R.D. Yu, 2009. Study on traffic accidents prediction model based on RBF neural network. Comput. Eng. Appl., 45: 188-190.

Sing, J.K., D.K. Basu, M. Nasipuri and M. Kundu, 2003. Improved $\mathrm{k}$-means algorithm in the design of $\mathrm{RBF}$ neural networks. Proceedings of the International Conference on Convergent Technologies for Asia-Pacific Region (TENCON 2003) Vol. 2, October 15-17, 2003, IEEE, Bangalore, India, pp: 841-845.

Sun, Y.P., L. Zhang and R. Lu, 2002. Tourist quantity forecast by using neural network. Hum. Geogr., 17: $50-52$.

Wei, H., 2005. Neural network structure design theory and method. National Defence Industry Press, Beijing, China.

Zhang, H. and J. Li, 2012. Prediction of tourist quantity based on RBF neural network. J. Comput., 7: 965-970. 